\title{
Direction of cross-modal information transfer affects human brain activation: a PET study
}

\author{
Ryuta Kawashima, ${ }^{1}$ Jobu Watanabe, ${ }^{2}$ Takashi Kato, ${ }^{3}$ Akinori Nakamura, ${ }^{3}$ Kentaro Hatano, ${ }^{3}$ Torsten Schormann, ${ }^{4}$ \\ Kazunori Sato, ${ }^{2}$ Hiroshi Fukuda, ${ }^{2}$ Kengo Ito ${ }^{3}$ and Karl Zilles ${ }^{4,5}$ \\ ${ }^{1} \mathrm{NICHe}$, Tohoku University, Aramaki, Aoba, Aobaku, Sendai 980-8579, Japan \\ ${ }^{2}$ IDAC, Tohoku University, Sendai, Japan \\ ${ }^{3}$ Department of Biofunctional Research, National Institute for Longevity Sciences, Obu, Japan \\ ${ }^{4}$ C. O. Vogt Institute of Brain Research, University of Duesseldorf, Duesseldorf, Germany \\ ${ }^{5}$ Institute of Medicine, Research Center Juelich, Germany
}

Keywords: inferior parietal lobule, premotor cortex, tactile information, visual information

\begin{abstract}
The purpose of this study was to determine the functional organization of the human brain involved in cross-modal discrimination between tactile and visual information. Regional cerebral blood flow was measured by positron emission tomography in nine right-handed volunteers during four discrimination tasks; tactile-tactile (TT), tactile-visual (TV), visual-tactile (VT), and visual-visual (VV). The subjects were asked either to look at digital cylinders of different diameters or to grasp the digital cylinders with the thumb and index finger of the right hand using haptic interfaces. Compared with the motor control task in which the subjects looked at and grasped cylinders of the same diameter, the right lateral prefrontal cortex and the right inferior parietal lobule were activated in all the four discrimination tasks. In addition, the dorsal premotor cortex, the ventral premotor cortex, and the inferior temporal cortex of the right hemisphere were activated during VT but not during TV. Our results suggest that the human brain mechanisms underlying cross-modal discrimination have two different pathways depending on the temporal order in which stimuli are presented.
\end{abstract}

\section{Introduction}

The term 'cross-modal' appears to have been introduced by Ettlinger (1960) in a study on cross-modal training in monkeys. That study (Ettlinger, 1960) marked the beginning of more systematic works on specific cross-modal performance, in nonhuman primates. Research interest in this area appears to have been further stimulated by the possibility that cross-modal performance abilities are of a higher order of cognitive complexity than those that involve only one modality; that is, cross-modal performance abilities may require more time to develop, and can only become established after the maturation of modality-specific systems. Despite extensive research on nonhuman primates and psychological research on infants, it remains unclear which specific brain structures and processes underlie such cross-modal performance abilities in normal human adults.

The earliest attempt to study the effects of brain lesions on crossmodal performance involved the use of the cross-modal recognition method of Cowey \& Weiskrantz (1975). Sahgal et al. (1975) and Petrides \& Iversen (1976) reported the impairment of cross-modal performance abilities following removal of the posterior temporal cortex and prestriate, or in the presence of lesions in the arcuate sulcus cortex. Impairment of abilities during a cross-modal recognition task was also observed in monkeys with lesions in the anterior cingulate and medial prefrontal cortex, but not in those with lesions in the lower lateral premotor cortex and areas PF and PFG of the parietal lobe (Aitken, 1980). Another study (Jarvis \& Ettlinger, 1977) used a

Correspondence: Professor Ryuta Kawashima, as above,

E-mail: ryuta@idac.tohoku.ac.jp

Received 23 January 2002, revised 25 April 2002, accepted 2 May 2002 different cross-modal recognition paradigm, in which monkeys learned a discrimination task in one modality (vision or touch), and performed trials in both presentation orders, namely, visual-to-tactile and tactile-to-visual. Lesions in the cortex, from the superior temporal cortex to the lateral prefrontal cortex of the monkeys, did not produce deficits, although the authors claimed that overtraining of the monkeys might have accounted for these negative results (Ettlinger \& Garcha, 1980). In general, however, lesion studies on monkeys have not yet provided consistent and convincing evidence that polysensory convergence areas mediate cross-modal performance. Ettlinger \& Wilson (1990) recently suggested an alternative model for the mechanism of cross-modal performance, claiming that, rather than the polysensory convergence areas mediating cross-modal matching, there is a so-called 'leakage' between perceptual/memory systems that were previously considered to be modality-specific. It was therefore suggested that modality-specific systems are not protected by impervious boundaries, and that representations built up by sensory events in one modality are still available for retrieval (although less efficiently) for input from another modality.

Recently, Hadjikhani \& Roland (1998) investigated the brain areas activated during tactile-visual cross-modal matching in which tactile and visual stimuli were presented simultaneously. In the present study, we determined the brain areas involved in cross-modal discrimination in two presentation orders, visual-to-tactile and tactile-to-visual, and compared these with regions activated during intramodal discrimination, in order to examine the extent to which the order of presentation of the cross-modal information affects brain regions involved in cross-modal discrimination. 


\section{Materials and methods}

\section{Subjects}

Nine healthy subjects, aged 20-30 years, participated in the study. All the subjects gave their informed consent according to the requirements of the Ethics Committee of the National Institute for Longevity Sciences. None had any history of grave medical illness and all had normal magnetic resonance images (MRIs). All the subjects were right-handed, as determined by the Edinburgh questionaire (Oldfield, 1971).

\section{Experimental procedure}

Prior to the positron emission tomography (PET) experiment, each subject had a catheter placed into the left brachial vein for tracer administration. The subjects were placed in a supine position on the bed of the PET scanner. The room was kept as quiet as possible. Each subject wore a head-mounted display (Eye-Trek; Olympus, Tokyo, Japan). Two haptic interfaces with fingerstalls (PHANTOM 1.5; Sensable Technology, MA, USA) were located beside the bed of the PET scanner, and the subjects were asked to insert the thumb and index finger of thier right hand into the fingerstalls of the haptic interfaces (Fig. 1a). These haptic interfaces were operated by software (GHOST; Sensable Technology, MA, USA) installed in a personal computer. This system enabled subjects to see and feel surfaces, and to grasp digital (virtual) three-dimensional (3D) objects. The subjects were able to use both texture gradient and binocular disparity as cues for 3D vision.

Eight cylinders of different diameters $(10,12,14,16,18,20,22$, and $24 \mathrm{~mm}$ ) but the same length $(50 \mathrm{~mm})$, smoothness and hardness were formed. These digital cylinders were always presented at the same location, that is, at the centre of the field of view, through the head-mounted display. These eight cylinders were used as visual stimuli in the discrimination tasks. The same series of eight cylinders were formed for use as tactile stimuli, but a white thin square board, $60 \times 60 \times 1 \mathrm{~mm}$ in size, was always presented in front of the cylinders (Fig. 1b). The subjects were able to touch the virtual cylinders by extending their hand behind the virtual board without interfering with it, but they could not directly see the cylinders. Prior to the PET experiment, the subjects were asked to look at and touch these cylinders to familiarize themselves with the virtual haptic system.

Each subject performed four discrimination tasks, two motor control tasks and one visual control task during the PET measurements (Table 1). Figure 1c shows an example of the sequence of the discrimination tasks. In the tactile-tactile discrimination task (TT), a sample tactile stimulus was presented for $5 \mathrm{~s}$ after the presentation of a 1-s cue signal (yellow circle: $10 \mathrm{~mm}$ in diameter). Then, a test tactile stimulus was presented for $5 \mathrm{~s}$ following a 1-s delay. During the delay period, a white 10 -mm-diameter circle was presented. The subjects were asked to grasp the digital cylinders behind the screen using their thumb and index finger, to move their fingertips several times up and down the surface of the cylinders, and to determine which of the cylinder had the largest diameter. They were instructed to say 'former' or 'latter' during the next cue period depending on whether they judged the sample or test tactile stimulus to be the largest, respectively. In this study, cylinders of different sizes were presented in each trial. The diameters of the sample and the test cylinders differed by $2 \mathrm{~mm}$, and the order of presentation was randomized. The task was continued for $2 \mathrm{~min}$

In the tactile-visual discrimination task (TV), a tactile stimulus was presented as sample stimulus, and the subjects were asked to estimate its diameter using their fingers. Then, a visual stimulus was

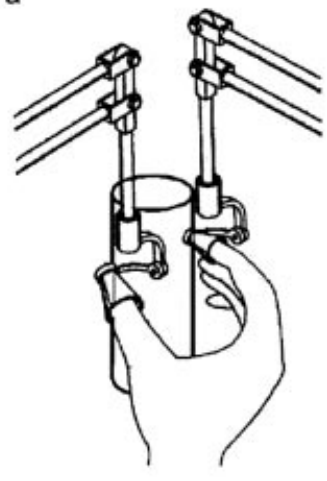

b

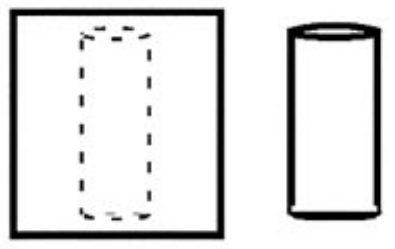

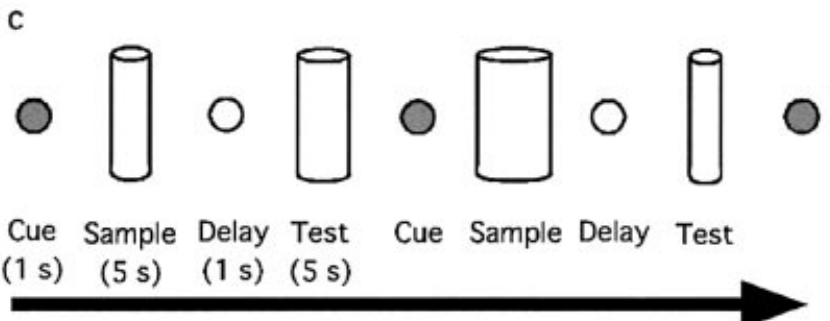

FIG. 1. (A) The subject inserting his thumb and index finger of the right hand into the fingerstalls of the haptic interfaces, and grasping a digital (virtual) cylinder. (B) Examples of visual and tactile stimuli. (C) An example of the sequence of discrimination tasks. The haptic systems enable subjects to see, feel surfaces, and grasp digital (virtual) 3D-objects. Subjects were able to use both texture gradient and binocular disparity as cues for 3D-vision.

presented as test stimulus, and the subjects were instructed to estimate the diameter of the cylinder visually, without grasping it. The other details were the same as those for the TT task. In the visual-tactile discrimination task (VT), visual and tactile stimuli were presented as sample and test stimuli, respectively. The other details were the same as those for the TV task. In the visual-visual discrimination task (VV), visual stimuli were presented as both sample and test stimuli. The subjects were instructed not to move their fingers throughout this task. The other details were the same as those for the TT task.

In motor control task 1 (MC1) and motor control task 2 (MC2), the task designs were the same as those in TT and TV, respectively, but a 16-mm-diameter cylinder was used as the lone stimulus. Therefore, the only difference between $\mathrm{MC} 1$ and $\mathrm{MC} 2$ was in the number of finger movements, that is, $\mathrm{MC} 1$ required twice as many finger movements as MC2. The subjects were asked to grasp the cylinders using their thumb and index finger and to move their fingertips several times up and down the surface of the cylinders when tactile stimuli were presented, or only to look at the visual stimuli without any finger movements. The subjects were instructed to say 'Yes' at the onset of the cue period. Prior to the start of these two tasks, the subjects were informed that the diameters of the cylinders to be presented as sample and test stimuli were exactly the same; therefore, they were not required to judge the difference in diameter between the two stimuli. In the visual control task (VC), the subjects were instructed not to move their fingers throughout this task. Visual stimuli were similar to those of the $\mathrm{VV}$, but only a 16-mm-diameter cylinder was used. The subjects were instructed to say 'Yes' at the onset of the cue period. Prior to the start of the task, the subjects were informed that the diameters of the cylinders were the same. The order 
TABLE 1. Sensory, motor, cognitive componenets of each task

\begin{tabular}{|c|c|c|c|c|c|c|}
\hline & \multicolumn{2}{|c|}{ Visual tasks } & \multicolumn{2}{|c|}{ Movements } & \multirow[b]{2}{*}{ Matching } & \multirow[b]{2}{*}{ Size of cylinder } \\
\hline & Sample & Test & Sample & Test & & \\
\hline VC & Cylinder & Screen & None & None & None & $16 \mathrm{~mm}$ fix \\
\hline MC1 & Screen & Screen & Tactile & Tactile & None & $16 \mathrm{~mm}$ fix \\
\hline $\mathrm{MC} 2$ & Screen & Cylinder & Tactile & None & None & $16 \mathrm{~mm}$ fix \\
\hline $\mathrm{TT}$ & Screen & Screen & Tactile & Tactile & Tactile-tactile & $10-25 \mathrm{~mm}$ random \\
\hline TV & Screen & Cylinder & Tactile & None & Tactile-visual & $10-25 \mathrm{~mm}$ random \\
\hline VT & Cylinder & Screen & None & Tactile & Visual-tactile & $10-25 \mathrm{~mm}$ random \\
\hline VV & Cylinder & Cylinder & None & None & Visual-visual & $10-25 \mathrm{~mm}$ random \\
\hline
\end{tabular}

of the tasks was counterbalanced across subjects. Finger movements during task performance were monitored and recorded by a digital video recorder. In addition, eye movements were monitored by an electrooculogram using surface electrodes.

\section{Data acquisition and analysis}

Regional cerebral blood flow was measured using a PET scanner (Siemens/CTI ECAT EXACT HR, Erlangen, Germany) (Wienhard et al., 1994). The emission scan was started immediately after administration of $12 \mathrm{mCi}(444 \mathrm{MBq}) \mathrm{H} 215 \mathrm{O}$ bolus injection, using the $3 \mathrm{D}$ collection mode. All tasks were started immediately after the bolus injection. Each PET measurement was commenced immediately after radioactive counts were determined on the PET monitor, and was continued for a period of $90 \mathrm{~s}$. In this study, one PET scan was obtained for each task. Each subject's MRI was anatomically normalized with respect to the standard brain anatomy of the Human Brain Atlas (Roland et al., 1994) by automated image registration (AIR) (Woods et al., 1998) and elastic transformation (Schormann et al., 1996). These parameters were subsequently used to transform each subject's PET image and MRI into the standard brain anatomy. Statistical parametric mapping (SPM96, Wellcome Department of Cognitive Neurology, London, UK) software was used for smoothing and statistical analysis (Friston et al., 1995). In this study, an isotropic Gaussian filter with an FWHM of $12 \mathrm{~mm}$ was used to increase the signal-to-noise ratio. Differences in global flow were covaried using ANCOVA. Comparisons across conditions were carried out by means of $t$-statistics, and thereafter transformed into normally distributed $Z$ statistics. In this study, comparisons were conducted for each motor control task vs. VC, TT vs. MC1, TV vs. MC2, VT vs. MC2, VV vs. $\mathrm{VC}$, and VT vs. TV. In addition, in order to determine brain areas showing polysensory activity, we employed a conjunction analysis (Price \& Friston, 1996), that is (TT - MC1) with (TV - MC2) with (VT - MC2) with (VV - VC) to determine brain activation common to all four discrimination tasks. For each comparison, the threshold for significant activation was set at $P<0.05$ (corrected for multiple comparisons). Finally, anatomical localization of the areas of activation in each comparison was carried out in relation to the mean anatomically standardized MRI.

\section{Results}

\section{Psychophysical measures}

The mean (SEM) percentages of correct responses during TT, TV, VT, and VV were 93.7 (2.7), 88.4 (4.4), 73.3 (3.3), and 98.8\% (1.2), respectively. The differences among the values were statistically significant $(F=12.8, P<0.00001$, ANOVA). The percentage of correct responses was the lowest during VT compared to that during the other
TABLE 2. Location of significant activation during motor control conditions compared with the visual control condition (VC)

\begin{tabular}{|c|c|c|c|c|c|}
\hline \multirow[b]{2}{*}{ Structure } & \multicolumn{3}{|c|}{ Coordinates (mm) } & \multicolumn{2}{|c|}{ Tasks ( $Z$-score) } \\
\hline & $x$ & $y$ & $z$ & MC1 & $\mathrm{MC} 2$ \\
\hline Sensori-motor (L) & -30 & -22 & 59 & 6.4 & 5.0 \\
\hline Premotor dorsal (R) & 29 & -10 & 60 & 4.7 & 4.0 \\
\hline Intraparietal (L) & -43 & -30 & 38 & 5.6 & 6.0 \\
\hline Inferior parietal (L) & -34 & -51 & 48 & 5.7 & 4.9 \\
\hline Inferior parietal (R) & 35 & -58 & 53 & 6.3 & 5.7 \\
\hline Cerebellum post erior(L) & -21 & -61 & -46 & 5.6 & 5.8 \\
\hline Cerebellum anterior (R) & 26 & -55 & -22 & 6.1 & 4.4 \\
\hline Cerebellum posterior (R) & 13 & -58 & -42 & 4.8 & 5.0 \\
\hline
\end{tabular}

Stereotaxic coordinates identify the location of the maxima of rCBF change corresponding to the atlas of Talairach \& Tournoux (1988). R, L, post, and ant indicate right, left, posterior, and anterior, respectively.

three discrimination tasks (paired $t$-test: VT vs. TT, $P<0.0001$; VT vs. TV, $P<0.05$; VT vs. VV, $P<0.000001$ ). No saccadic, pursuit, or slow eye movements were observed during each task.

\section{Brain activation}

Regions exhibiting significant activation during the motor control tasks ( $\mathrm{MC} 1$ and $\mathrm{MC} 2$ ) compared with those during $\mathrm{VC}$ are presented in Table 2. Significant brain activations determined by TT minus $\mathrm{MC} 1, \mathrm{TV}$ minus MC2, VT minus MC2, VV minus VC, and VT vs. TV comparisons are summarized in Table 3.

\section{Premotor cortex}

Three different task-related activation foci were found in the premotor cortex of the right hemisphere; one in the dorsal part, and two in the ventral part. The right dorsal premotor cortex was significantly activated as determined by the VT minus MC2 comparison (Fig. 2). This area showed increases in regional cerebral blood flow (rCBF) during TT, but not during TV or VV (Fig. 3). An area in the right ventral premotor cortex was specifically activated as determined by the VT minus MC2 comparison (Fig. 2), but was not activated during TV, TT or VV (Fig. 3). Another area in the ventral premotor cortex located posterior to the former VT-specific area was found to be significantly activated as determined by the TT vs. MC1 comparison (Fig. 2). This region showed increases in $\mathrm{rCBF}$ during TV and VT, but not during VV (Fig. 3).

\section{Prefrontal cortex}

An area in the right middle frontal gyrus was significantly activated as determined by TT minus MC 1, TV minus MC2, and VT minus MC2 
TABLE 3. Significant activation during discrimination tasks

\begin{tabular}{|c|c|c|c|c|}
\hline \multirow[b]{2}{*}{ Structure } & \multicolumn{3}{|c|}{ Coordinates $(\mathrm{mm})$} & \multirow[b]{2}{*}{$Z$-score } \\
\hline & $x$ & $y$ & $z$ & \\
\hline \multicolumn{5}{|l|}{ (TT - MC1) } \\
\hline Premotor ventral (R) & 46 & -1 & 32 & 4.6 \\
\hline Middle Frontal (R) & 32 & 49 & 4 & 4.1 \\
\hline Inferior Parietal (R) & 32 & -53 & 34 & 5.2 \\
\hline \multicolumn{5}{|l|}{$(\mathrm{TV}-\mathrm{MC} 2)$} \\
\hline Middle Frontal (R) & 32 & 52 & 8 & 4.1 \\
\hline Inferior Parietal (R) & 44 & -52 & 36 & 4.6 \\
\hline \multicolumn{5}{|l|}{$(\mathrm{VT}-\mathrm{MC} 2)$} \\
\hline Premotor dorsal (R) & 28 & -1 & 48 & 4.8 \\
\hline Premotor ventral (R) & 42 & 14 & 30 & 4.0 \\
\hline Middle Frontal (R) & 32 & 46 & 0 & 4.6 \\
\hline Inferior Parietal (R) & 36 & -56 & 34 & 5.1 \\
\hline Inferior Temporal (R) & 56 & -33 & -6 & 4.2 \\
\hline \multicolumn{5}{|l|}{$(\mathrm{VV}-\mathrm{VC})$} \\
\hline Inferior Parietal (R) & 41 & -61 & 42 & 4.5 \\
\hline Inferior Temporal (R) & 63 & -14 & -2 & 4.2 \\
\hline \multicolumn{5}{|l|}{$(\mathrm{VT}-\mathrm{TV})$} \\
\hline Premotor ventral (R) & 46 & 16 & 27 & 4.8 \\
\hline
\end{tabular}

See Table 2 for details.

comparisons (Fig. 2). VV also induced increases in $\mathrm{rCBF}$ in this area when compared with VC (Fig. 3). Thus, this area in the right middle frontal gyrus was involved in all of the four discrimination tasks.

\section{Parietal cortex}

Two different task-related activation foci were found in the inferior parietal lobule of the right hemisphere; one in the angular gyrus, and the other in the supramarginal gyrus. An area in the angular gyrus was significantly activated as determined by the VV minus VC comparison (Fig. 2). This area showed increases in rCBF during VT (Fig. 3), but not during TT or TV. The inferior parietal lobule was significantly activated as shown by TT minus MC1, TV minus MC2 as well as VT minus MC2 comparisons (Fig. 3), but was not activated during VV (Fig. 3).

\section{Temporal Cortex}

An area in the right inferior temporal sulcus was significantly activated as shown by the VT minus MC2, and VV minus VC comparisons (Fig. 2), but was not activated during TT and TV (Fig. 3).

Direct comparison between VT and TV showed a significant activation in the right ventral premotor cortex in the VT minus TV comparison. No significant activation was found in the TV minus VT comparison.

The conjunction analysis revealing brain activations common to all four discrimination tasks showed significant activation in an area of the right prefrontal cortex. The volume of the activated area was $2584 \mathrm{~mm}^{3}$.

In summary, TV was found to activate certain brain areas, parts of which were also activated by TT and VT. On the other hand, VT activated the same brain areas activated by TT and/or VV, as well as a specific area in the right ventral premotor area (Fig. 4).

\section{Discussion}

In this study, we determined the brain regions that were activated during both cross-modal and intramodal size discrimination. The
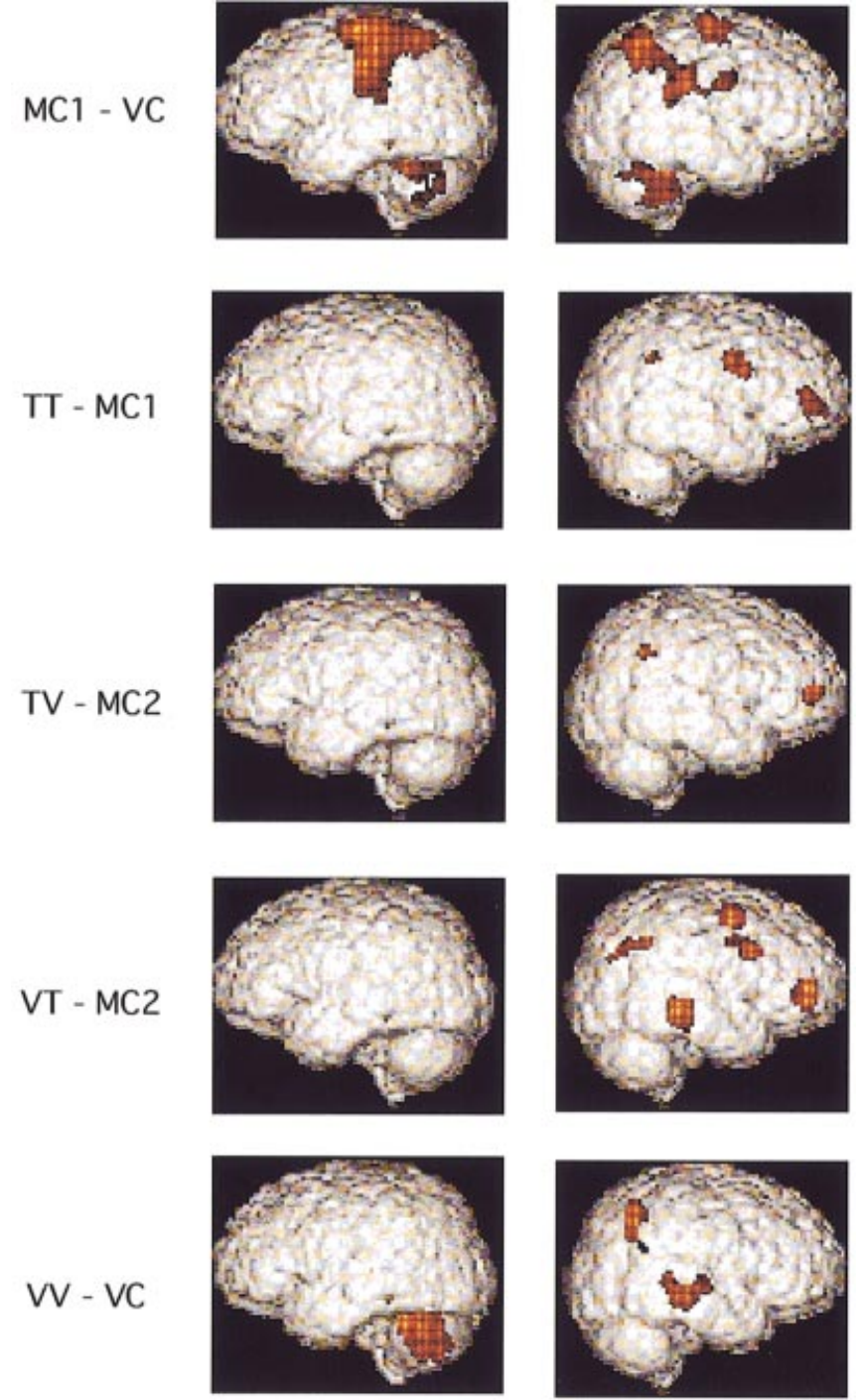

FIG. 2. Surface projections of colour-coded SPMs superimposed onto stereotactically normalized T1-weighted MRI human brain atlas (Roland et al., 1994) showing significant activation.

most notable finding was that the activation patterns during visualtactile and tactile-visual cross-modal discrimination were different. We found that when working memory for tactile information was activated during visual information processing (tactile-visual discrimination: TV), only a few of the brain areas involved in intramodal discrimination (modality-specific areas) were activated. On the other hand, when working memory for visual information was activated during tactile information processing (visual-tactile discrimination: VT), there was activation, not only of several modality-specific areas but also a specific area for cross-modal discrimination in the ventral premotor cortex. These results indicate that human brain mechanisms underlying cross-modal (visual and somatosensory) discrimination involve two different pathways depending on the temporal order of stimulus presentation.

\section{Cross-modal transfer}

Psychophysical measurement of the subject's performance indicates that the cross-modal transfer of information was inaccurate during VT compared with TV. In addition, more brain areas were activated 

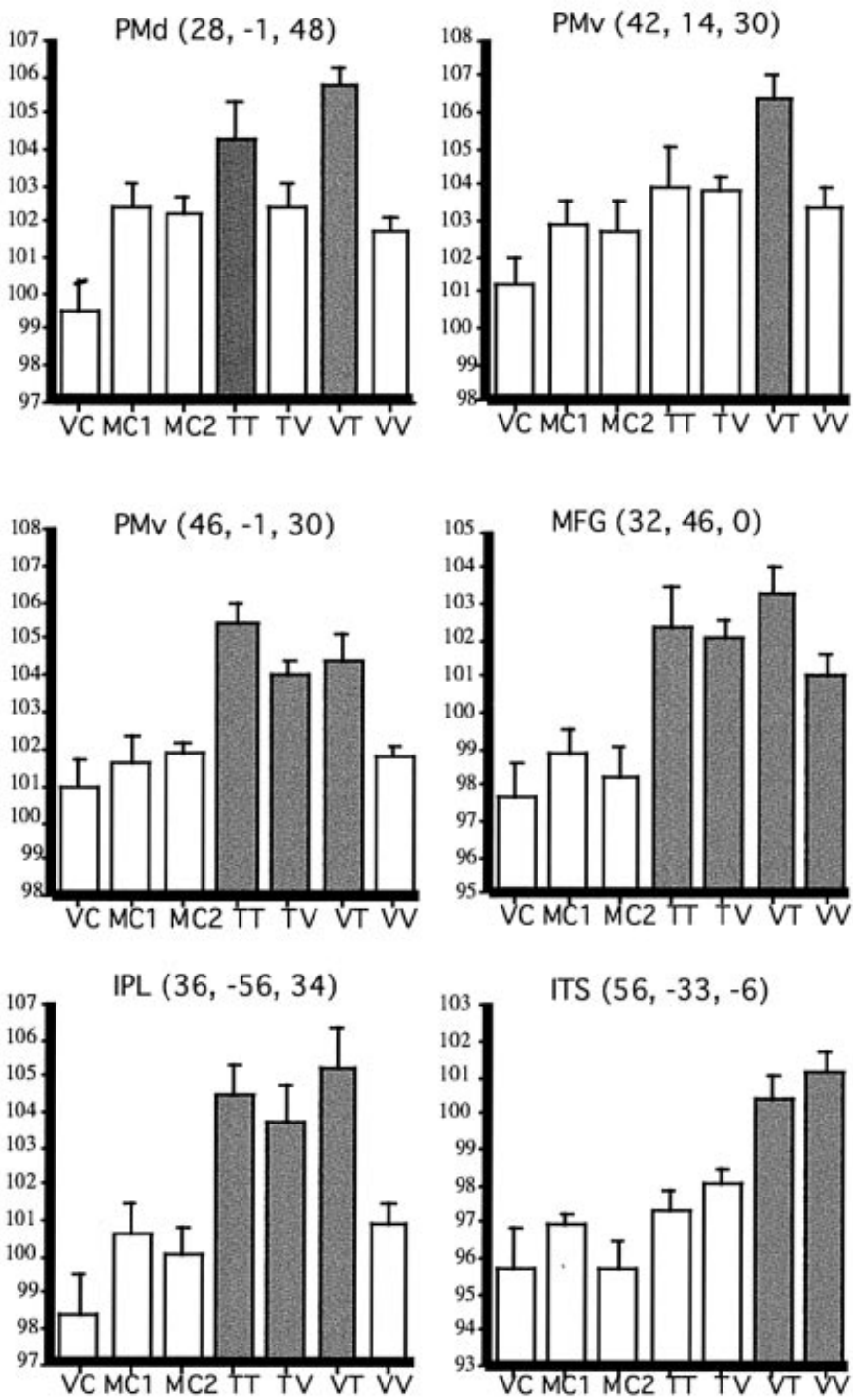

FIG. 3. Examples of adjusted mean normalized activity during each task show voxels with peak activation. Error bars indicate SD.

during VT than during TV. Our results are consistent with those of previous psychological studies of infants and children indicating that tactile-visual transfer is easier than visual-tactile transfer (Molander \& Garvill, 1981; Juurmma \& Lehtinen-Railo, 1988; Rose \& Orlian, 1991).

Our results are also consistent, to a certain degree, with those of a previous monkey lesion study by Streicher \& Ettlinger (1987), suggesting that these cross-modal operations are not symmetrical. These authors examined cross-modal performance using entirely new and unfamiliar objects as stimuli. With lesions in the frontal, temporal and parietal polysensory cortices, there was impairment in crossmodal recognition of unfamiliar objects, despite normal performance when familiar objects were used. The effects of these lesions may also have been more pronounced in visual-to-tactile tasks than in tactile-to-visual tasks. The authors suggested that the neocortical areas are essential for the formation of new representations during visual learning if they are needed immediately after tactile recognition tasks, but that they are not necessary for refreshing previously formed visual representations. Alternatively, the neocortex is
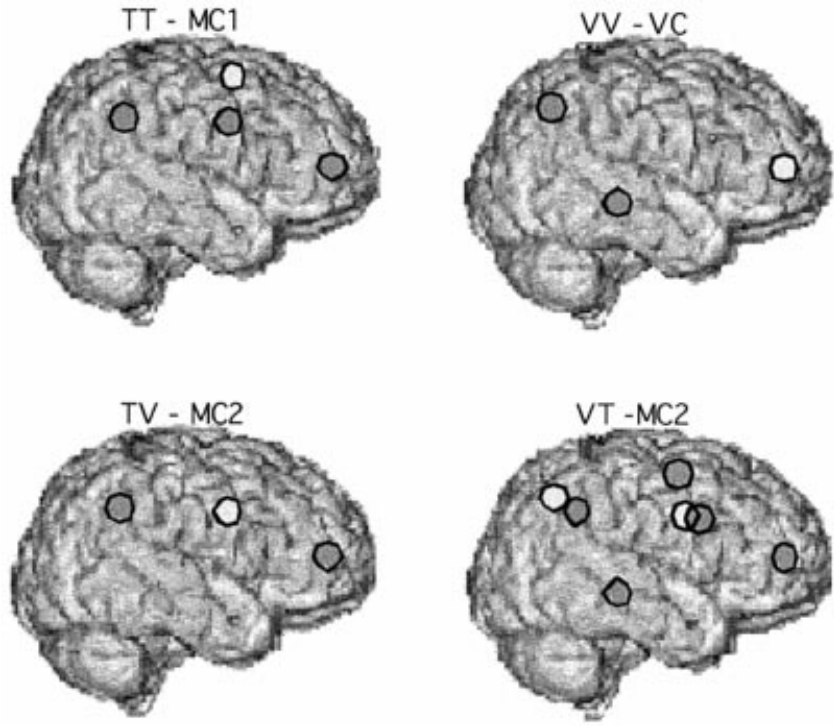

FIG. 4. Activation patterns for the right hemisphere. Gray circles indicate areas of statistically significant activation. White circles indicate areas showing increases in $\mathrm{rCBF}$, but which failed to reach a significant increase.

probably necessary for predicting qualities of a tactile object based on previous visual information. Therefore, our results indicate that different cognitive operations may be performed in response to the same stimuli in VT vs. TV. Thus, a greater cognitive load may be required for performing $\mathrm{VT}$ than $\mathrm{TV}$, and human brain activity involved in cross-modal transfer differs in accordance with the direction of transfer.

Another possibility is that the difference in activation patterns between TV and VT may be induced by the difference in task difficulty, since the mean percentage of correct responses during VT was lower than that during TV. However, recent brain imaging studies have shown that an increase in task difficulty is associated with an increase in regional cerebral activity only in brain areas that are involved in specific tasks, particularly the prefrontal cortex and/or the anterior cingulate cortex (Baker et al., 1996; Barch et al., 1997; Gerlach et al., 1999; Speck et al., 2000; Sunaert et al., 2000). To our knowledge, there is no report on the activation of additional brain area(s) in relation to increased task difficulty.

\section{Prefrontal cortex}

An area in the lateral prefrontal cortex located in the right middle frontal gyrus was consistently activated during TT, TV, and VT. This area also showed increased activity during VV. Although the location of the peak activation differed slightly, the activated areas overlapped among these tasks. This activation probably represents commonalities in all the discrimination tasks. Discrimination tasks used in this study were delayed tasks that required working memory of an internal representation of the first stimulus, that is, the subjects had to keep stimulus information in their working memory in order to discriminate it from another set of stimuli. Experimental studies of nonhuman primates have shown the crucial role played by the prefrontal cortex in maintaining working memory (Goldman-Rakic, 1987, 1995 for review). In previous neuroimaging studies of humans, the lateral prefrontal area of the right hemisphere was activated during both spatial (Smith et al., 1995) and nonspatial working memory tasks (Petrides et al., 1993; Smith et al., 1995; Klingberg et al., 1996; Kawashima et al., 1998). We could not make a simple 
comparison of our results with those of other neuroimaging studies, as different laboratories use different systems to transform their neuroimaging data to their standard anatomical space. Nevertheless, there is good agreement between our results and those of other neuroimaging studies, in that the prefrontal cortex activation in the present study has almost the same stereotaxic coordinates as those described in other activation studies. Therefore, the most probable interpretation is that the prefrontal cortex activations seen in the present study were related to working memory.

\section{Premotor cortex}

In this study, three different task-related activation foci were found in the premotor cortex; one in the dorsal part, and two in the ventral part. A region in the right ventral premotor cortex was specifically activated during VT, but not during TV, TT or VV. Therefore, this area was activated when working memory for tactile information was activated during visual information processing. One possible interpretation is that this activation was due to a process in which visual information was transformed to an internal hand motor pattern to be compared with a hand motor pattern during haptic stimulation. Based on results of neurophysiological studies of monkeys, it is known that the activity of neurons in the ventral premotor cortex is related to the visual inputs (Rizzolatti et al., 1988; Boussaoud et al., 1996).

In the present study, a region in the dorsal premotor cortex was activated during TT and VT, but not during TV and VV. Although $\mathrm{TV}$, which required tactile information processing, did not activate this area, it could be specifically activated when working memory of visual information was activated during tactile information processing. In humans, the premotor cortex is known to be involved in verbal or visuospatial working memory (Smith \& Jonides, 1998, 1999 for review), and to our knowledge, there are no studies showing involvement of the premotor cortex in tactile working memory. Another region in the ventral premotor cortex located posterior to the VT-specific area was activated during TT, TV and VT but not during VV. Therefore, the activation of this ventral premotor cortex is thought to be related to tactile information processing. In monkeys, neurons in the ventral and dorsal premotor cortices are known to be fired in relation to the processing of somatosensory stimuli (Rizzolatti et al., 1981; Kurata \& Tanji, 1986). Consistent results were reported in previous human PET studies indicating the involvement of the premotor cortex in tactile perception (Bottini et al., 1995; Burton et al., 1999; Mima et al., 1999). O'Sullivan et al. (1994) also suggested that the right dorsal premotor cortex is involved in length discrimination but not in roughness discrimination. It is therefore possible that the two regions in the premotor cortex activated in this study comprise the cortical network involved in tactile macrogeometric information processing.

\section{Inferior parietal cortex}

In this study, an area in the inferior parietal lobule showed activation during TT, TV and VT but not during VV, indicating that this area was involved in tactile information processing. This area was located anterior to the inferior parietal lobule, which was determined by the VV vs. VC comparison. Therefore, we identified two different foci in the inferior parietal cortex which were involved in discrimination tasks. The anterior focus, which was activated during TT, TV and VT, was located in the supramarginal gyrus; on the other hand, the posterior focus, which was activated during $\mathrm{VV}$, was located in the angular gyrus. These results are consistent with those reported in previous PET studies indicating that the activation of the supramarginal gyrus is related to the somatosensory discrimination of length (O'Sullivan et al., 1994) and shape (Hadjikhani \& Roland,
1998; Roland et al., 1998), and that the angular gyrus is involved in visual discrimination of shape (Hadjikhani \& Roland, 1998) and size (Faillenot et al., 1999). Our results are also consistent with those of human lesion studies indicating that right posterior parietal cortex lesions are associated with the neglect of visual and somatosensory stimuli located in an extrapersonal space (Mesulam, 1981; Posner et al., 1984). It should be noted that a similar activation pattern of this supramarginal area was observed in a part of the ventral premotor cortex (Fig. 2). A similar parietal-premotor cortex activation pattern has been reported in a recent fMRI study of tactile exploration (Binkofski et al., 1999). In monkeys, the anterior part of the posterior parietal lobule is interconnected with the ventral premotor cortex (Cavada \& Goldman-Rakic, 1989). Therefore, we suggest that the coactivation of the supramarginal gyrus and the ventral premotor cortex indicates that these two areas are part of the cortical network involved in tactile information processing.

Another possible interpretation of this inferior parietal cortex activation is that this area may also be involved in processing representations of objects and space in the somatosensory modality, as suggested by neuropsychological studies (Iwamura \& Tanaka, 1996; Duhamel et al., 1998).

\section{Inferior temporal cortex}

In this study, an area in the cortex lining the inferior temporal sulcus was activated during VT and VV but not during TT and TV. This activity may well be related to visual working memory, since TV, in which subjects discriminate tactile information in working memory from presented visual information, did not activate this area. The inferior temporal cortex has been recognized as one of the final stages of the visual form pathway, which leads from the primary visual cortex (V1) to V2 and V4 (Ungerleider \& Mishkin, 1982). Neurophysiological studies of monkeys have demonstrated that this region is involved in visual pattern discrimination and in recognition of visual objects (Miyashita, 1993 for review), as well as in visual working memory (Miller \& Desimone, 1991; Miller et al., 1993; Chelazzi et al., 1998). It is also important to note that neurons selective for $3 \mathrm{D}$ shape exist in the inferior temporal cortex of Macaque (Janssen et al., 2000). In humans, brain imaging studies have shown activation of the cortex lining the inferior temporal sulcus during tasks requiring a nonspatial visual working memory of objects (Roland \& Gulyas, 1995; Smith et al., 1995; Kawashima et al., 1998; Gerlach et al., 1999; Postle et al., 2000; Faillenot et al., 2001). Thus, our results together with results of these previous studies indicate that the inferior temporal cortex activation observed in this study might be related to the visual working memory of 3D shape.

\section{Acknowledgements}

This study was supported in part by a JSPS-RFTF (97L00202) grant, a Grantin-Aid for Scientific Research on Priority Areas (C) - Advanced Brain Science Project- from Ministry of Education, Science, Sports and Culture, a research grant from Telecommunication Advancement Organization of Japan, and Basic Research 21 for Breakthroughs in Info-Communications, and an SFB 194/A6 grant to K.Z.

\section{Abbreviations}

MC, motor control task; MRI, magnetic resonance imaging; PET, positron emission tomography; rCBF, regional cerebral blood flow; SPM, Statistical parametric mapping; TT, tactile-tactile task; TV, tactile-visual task; VC, visual control task; VT, visual-tactile task; VV, visual-visual task. 


\section{References}

Aitken, P.G. (1980) Lesion effects on tactual to visual cross-modal matching in the rhesus monkey. Neuropsychologia, 18, 575-578.

Baker, S.C., Rogers, R.D., Owen, A.M., Frith, C.D., Dolan, R.J., Frackowiak, R.S. \& Robbins, T.W. (1996) Neural systems engaged by planning: a PET study of the Tower of London task. Neuropsychologia, 34, 515-526.

Barch, D.M., Braver, T.S., Nystrom, L.E., Forman, S.D., Noll. D.C. \& Cohen, J.D. (1997) Dissociating working memory from task difficulty in human prefrontal cortex. Neuropsychologia, 35, 1373-1380.

Binkofski, F., Buccino, G., Posse, S., Seitz, R.J., Rizzolatti, G. \& Fruend, H. (1999) A fronto-parietal circuit for object manipulation in man: evidence from an fMRI-study. Eur. J. Neurosci., 11, 3276-3286.

Bottini, G., Paulesu, E., Sterzi, R., Warburton, E., Wise, R.J., Vallar, G., Frackowiak, R.S. \& Frith, C.D. (1995) Modulation of conscious experience by peripheral sensory stimuli. Naure, 376, 778-781.

Boussaoud, D., di Pellegrino, G. \& Wise, S.P. (1996) Frontal mechanisms subserving vision-for-action versus vision-for-perception. Behav. Brain Res., 72, 1-15.

Burton, H., Abend, N.S., MacLeod, A.M.K., Sinclair, R.J., Snyder, A.Z. \& Raichle, M.E. (1999) Tactile attention tasks enhance activation in somatosensory regions of parietal cortex: a positron emission tomography study. Cereb. Cortex, 9, 662-674.

Cavada, C. \& Goldman-Rakic, P.S. (1989) Posterior parietal cortex in rhesus monkey. I. Parcellation of areas based on distinctive limbic and sensory corticocortical connections. J. Comp. Neurol., 287, 393-421.

Chelazzi, L., Duncan, J., Miller, E.K. \& Desimone, R. (1998) Responses of neurons in inferior temporal cortex during memory-guided visual search. J. Neurophysiol., 80, 2918-2940.

Cowey, A. \& Weiskrantz, L. (1975) Demonstration of cross-modal matching in rhesus monkeys, Macaca mulatta. Neuropsychol., 13, 117-120.

Duhamel, J.R., Colby, C.L. \& Goldgerb, M.E. (1998) Ventral intraparietal area of the macaque: congruent visual and somatic response properties. J. Neurophysiol., 79, 126-136.

Ettlinger, G. (1960) Cross-modal transfer of training in monkeys. Behaviour 16, 56-65.

Ettlinger, G. \& Garcha, H.S. (1980) Cross-modal recognition by the monkey: the effects of cortical removals. Neuropsychology, 18, 685-692.

Ettlinger, G. \& Wilson, W.A. (1990) Cross-modal performance: behavioral processes, phylogenetic considerations and neural mechanisms. Behav. Brain Res., 40, 169-192.

Faillenot, I., Decety, J. \& Jeannerod, M. (1999) Human brain activity related to the perception of spatial features of objects. Neuroimage, 10, 114-124.

Faillenot, I., Sunaert, S., Van Hecke, P. \& Orban, G.A. (2001) Orientation discrimination of objects and gratings compared: an fMRI study. Eur. $J$. Neurosci., 13, 585-596.

Friston, K.J., Holmes, A.P., Worsley, K.J., Poline, J.-P., Frith, C.D. \& Frackowiak, R.S.J. (1995) Statistical parametric maps in functional imaging: a general linear approach. Hum. Brain Map., 2, 189-210.

Gerlach, C., Law, I., Gade, A. \& Paulson, O.B. (1999) Perceptual differentiation and category effects in normal object recognition: a PET study. Brain, 122, 2159-2170.

Goldman-Rakic, P.S. (1987) Circuitry of primate prefrontal cortex and regulation of behavior by representational memory. In Plum, F. (ed.), Handbook of physiology Sec 1, The nervous system, V. Higher Functions of the Brain. American Physiology Association, Bethesda, pp. 373-417.

Goldman-Rakic, P.S. (1995) Architecture of the prefrontal cortex and the central executive. Ann. NY Acad. Sci., 769, 71-83.

Hadjikhani, N. \& Roland, P.E. (1998) Cross-modal transfer of information between the tactile and the visual representations in the human brain: a positron emission tomographic study. J. Neurosci., 18, 1072-1084.

Iwamura, Y. \& Tanaka, M. (1996) Representation of reaching and grasping in the monkey postcentral gyrus. Neurosci. Lett., 214, 147-150.

Janssen, P., Vogels, R. \& Orban, G.A. (2000) Selectivity for 3D shape that reveals distinct areas within macaque inferior temporal cortex. Science, $\mathbf{2 8 8}$, 2054-2056.

Jarvis, M.J. \& Ettlinger, G. (1977) Cross-modal recognition in chimpanzees and monkeys. Neuropsychology, 15, 499-506.

Juurmma, J. \& Lehtinen-Railo, S. (1988) Cross-modal transfer of forms between vision and touch. Scand. J. Psychol., 29, 95-110.

Kawashima, R., Satoh, K., Goto, R., Inoue, K., Itoh, M. \& Fukuda, H. (1998) The role of the left inferior temporal cortex for visual pattern discrimination - a PET study. Neuroimage, 9, 1581-1586.

Klingberg, T., Kawashima, R. \& Roland, P.E. (1996) Activation of multimodal cortical areas underlies short-term memory. Eur. J. Neurosci., 8, 1965-1971.
Kurata, K. \& Tanji, J. (1986) Premotor cortex neurons in macaques: activity before distal and proximal forelimb movements. J. Neurosci., 6, 403-411.

Mesulam, M.M. (1981) A cortical network for directed attention and unilateral neglect. Ann. Neurol., 10, 309-325.

Miller, E.K. \& Desimone, R. (1991) A neural mechanism for working and recognition memory in inferior temporal cortex. Science, 254, 1377-1379.

Miller, E.K., Li, L. \& Desimone, R. (1993) Activity of neurons in anterior inferior temporal cortex during a short-term memory task. J. Neurosci., 13, $1460-1478$.

Mima, T., Sadato, N., Yazawa, S., Hanakawa, T., Fukuyama, H., Yonekura, Y. \& Shibasaki, H. (1999) Brain structures related to active and passive finger movements in man. Brain, 122, 1989-1997.

Miyashita, Y. (1993) Inferior temporal cortex: where visual perception meets memory. Аnпи. Rev. Neurosci., 16, 245-263.

Molander, B. \& Garvill, J. (1981) The invariance of asymmetric cross-modal transfer effects. Scand. J. Psychol., 20, 171-177.

O'Sullivan, B.T., Roland, P.E. \& Kawashima, R. (1994) A PET study of somatosensory discrimination in man -microgeometry versus macrogeometry-. Eur. J. Neurosci., 6, 137-148.

Oldfield, R.C. (1971) The assessment and analysis of handedness: the Edinburgh inventory. Neuropsychology, 9, 97-113.

Petrides, M., Alivisatos, B., Evans, A.C. \& Meyer, E. (1993) Dissociation of human mid-dorsolateral from posterior dorsolateral frontal cortex in memory processing. Proc. Natl. Acad. Sci. USA, 90, 873-877.

Petrides, M. \& Iversen, S.D. (1976) Cross-modal matching and the primate frontal cortex. Science, 192, 1023-1024.

Posner, M.I., Walker, J.A., Friedrech, F.J. \& Rafal, R.D. (1984) Effects of parietal injury on covert orientation of attention. J. Neurosci., 4, 1863-1874.

Postle, B.R., Stern, C.E., Rosen, B.R. \& Corkin, S. (2000) An fMRI investigation of cortical contribution to spatial and nonspatial visual working memory. Neuroimage, 11, 447-450.

Price, C.J. \& Friston, K.J. (1996) Cognitive conjunction: a new approach to brain activation experiments. Neuroimage, 5, 261-270.

Rizzolatti, G., Camarda, L., Fogassi, L., Gentilucci, M., Luppino, G. \& Matelli, M. (1988) Functional organization of inferior area 6 in the macaque monkey. II. Area F5 and the control of distal movements. Exp. Brain Res., 71, 491-507.

Rizzolatti, G., Scandolara, C., Matelli, M. \& Gentilucci, M. (1981) Afferent properties of prearcuate neurons in macaque monkey. I. Somatosensory responses. Behav. Brain Res., 2, 125-146.

Roland, P.E., Graufelds, C.J., Wahlin, J., Ingelman, L., Andersson, M., Ledberg, A., Pedersen, J., Akerman, S., Dabringhaus, A. \& Zilles, K. (1994) Human brain atlas: For high resolution functional and anatomical mapping. Hum. Brain Map., 1, 173-184.

Roland, P.E. \& Gulyas, B. (1995) Visual memory, visual imagery, and visual recognition of large field patterns by the human brain: functional anatomy by positron emission tomography. Cereb. Cortex, 5, 79-93.

Roland, P.E., O'Sullivan, B.T. \& Kawashima, R. (1998) Shape and roughness activate different somatosensory areas in the human brain. Proc. Natl. Acad. Sci. USA, 95, 3295-3300.

Rose, S.A. \& Orlian, E.K. (1991) Asymmetries in infant cross-modal transfer. Child. Dev., 62, 706-718.

Sahgal, A., Petrides, M. \& Iversen, S.D. (1975) Cross-modal matching in the monkey after discrete temporal lobe lesions. Nature, 257, 672-674.

Schormann, T., Henn, S. \& Zilles, K. (1996) A new approach to fast elastic alignment with application to human brain. Lecture Notes Comput. Sci. 1131, 337-342.

Smith, E.E. \& Jonides, J. (1998) Neuroimaging analyses of human working memory. Proc. Natl. Acad. Sci. USA, 95, 12061-12068.

Smith, E.E. \& Jonides, J. (1999) Storage and executive processes in the frontal lobes. Science, 283, 1657-1661.

Smith, E.E., Jonides, J., Koeppe, R.A., Awh, E., Schumacher, E.H. \& Minoshima, S. (1995) Spatial versus object working memory: PET Investigations. J. Cogn. Neurosci., 7, 337-356.

Speck, O., Ernst, T., Braun, J., Koch, C., Miller, E. \& Chang, L. (2000) Gender differences in the functional organization of the brain for working memory. Neuroreport., 11, 2581-2585.

Streicher, M. \& Ettlinger, G. (1987) Cross-modal recognition of familiar and unfamiliar objects by the monkey: the effects of ablation of polysensory neocortex or of the amygdaloid complex. Behav. Brain Res., 23, 95-107.

Sunaert, S., Van Hecke, P., Marchal, G. \& Orban, G.A. (2000) Attention to speed of motion, speed discrimination, and task difficulty: an fMRI study. Neuroimage., 11, 612-623.

Talairach, J. \& Tournoux, P. (1988) Co-Planar Stereotaxic Atlas of the Human Brain. George Thieme Verlag, Stuttgart. 
144 R. Kawashima et al.

Ungerleider, L.G. \& Mishkin, M. (1982) Two cortical visual pathways for object recognition and spatial perception. In Changas, C., Gattass, R. \& Gross, C.G. (eds), Pattern Recognition Mechanisms. Pontifical Academy of Sciences, Vatican City, pp. 549-586.

Wienhard, K., Dahibom, M., Eriksson, L., Michel, C., Bruckbauer, T., Pietzyk,
U. \& Heiss, W.D. (1994) The ECAT EXACT HR: performance of a new high resolution positron scanner. J. Comput. Assist. Tomogr., 18, 110-118. Woods, R.P., Grafton, S.T., Watson, J.D.G., Sicotte, N.L. \& Mazziotta, J.C. (1998) Automated image registration. II. Intersubject validation of linear and nonlinear methods. J. Comput. Assist. Tomogr., 22, 153-165. 